\title{
A SEPARABLE BANACH SPACE WITH THE RADON-NIKODÝM PROPERTY THAT IS NOT ISOMORPHIC TO A SUBSPACE OF A SEPARABLE DUAL
}

\author{
PHILIP W. MCCARTNEY AND RICHARD C. O'BRIEN
}

\begin{abstract}
In this paper an example is given of a separable Banach space with the Radon-Nikodym property that is not isomorphic to a subspace of a separable dual space.
\end{abstract}

In [5] Uhl showed that if every separable closed linear subspace of a Banach space $X$ is isomorphic to a subspace of a separable dual, then $X$ has the Radon-Nikodým property (RNP). In that same paper, he asked whether every separable Banach space $X$ with the RNP is isomorphic to a subspace of a separable dual space. Three years later, in 1975, Stegall [4] showed that if $X$ is a subspace of a dual space, then the answer to Uhl's question is yes. We present in this note an example which shows that for general Banach spaces the answer is no.

An example of a separable Banach space that has the RNP and is not isometric (but is isomorphic) to a subspace of a separable dual is given in [2] and [3]. Our construction employs this example as well as other results obtained in these papers. We will state the definitions and results to be used in this note and refer the reader to [2] for the details.

Let $X$ be a Banach space and let $\varepsilon$ be a positive number. Let $S=\{(k, j)$ : $\left.k \in \mathbf{N}, j \in \mathbf{N}, 1 \leqslant j \leqslant 2^{k-1}\right\}$, and $S_{n}=\{(k, j) \in S: k \leqslant n\}$ for each $n \in \mathbf{N}$. A tree with separation constant $\varepsilon$ is a function $T: S \rightarrow X$ such that for every $(k, j)$ in $S$

(i) $T(k, j)=\frac{1}{2}[T(k+1,2 j)+T(k+1,2 j-1)]$,

(ii) $\|T(k, j)\| \leqslant 1$,

(iii) $\|T(k+1,2 j)-T(k+1,2 j-1)\|>\varepsilon$.

A finite tree with separation constant $\varepsilon$ is a function $T: S_{n} \rightarrow X$ that satisfies conditions (i), (ii) and (iii). It is known that if a Banach space $X$ contains a tree, then $X$ does not have the Radon-Nikodým property [1, p. 127]. The Banach space $X$ is said to have the $(\varepsilon, \delta)$-neighborly tree property (NTP) if there are positive numbers $\varepsilon$ and $\delta$ for which $\delta<\varepsilon / 4$ and the closed unit ball of $X$ contains a sequence $\left(T_{n}\right)$ of finite trees satisfying:

(i) Each $T_{n}$ is defined on $S_{n}$.

(ii) Each $T_{n}$ has separation constant $\varepsilon$.

Presented to the Society October 16, 1978; received by the editors January 25, 1979.

AMS (MOS) subject classifications (1970). Primary 46B99; Secondary 46G05.

Key words and phrases. Radon-Nikodým property, tree, neighborly tree property, separable dual space. 
(iii) For every $(k, j)$ in $S$, there is a ball in $X$ of radius $\delta$ that contains $\left\{x_{n}^{k, j}\right.$ : $n>k\}$ where $T_{n}(k, j)=x_{n}^{k, j}$ when $(k, j) \in S_{n}$.

The number $\delta$ is called a neighborly constant.

The neighborly tree property was introduced in [2] where it was shown that any dual space that has the neighborly tree property contains a tree [Theorem 6] and hence does not have the Radon-Nikodým property. In the same paper it was shown that for any positive $\delta<\frac{1}{2}$ there exists a separable Banach space $X_{\delta}$ that has the RNP and has the $(2, \delta)$-NTP. These spaces are used in the construction of the example presented in this note, but before giving that construction we discuss the behavior of the $(\varepsilon, \delta)$-NTP under isomorphisms.

Suppose that $\varphi: X \rightarrow Z$ is an isomorphism of $X$ into another Banach space $Z$ and that $c$ is a positive number such that for all $x$ in $X,(1 / c)\|x\| \leqslant\|\varphi(x)\|$ $\left\langle c\|x\|\right.$. If $\left(T_{n}\right)$ is an $(\varepsilon, \delta)$ neighborly tree structure in $X$, then it is easily seen that $\left((1 / c) \varphi\left(T_{n}\right)\right)$ satisfies all the conditions for a neighborly tree structure in $Z$ with separation constant $\varepsilon / c^{2}$ and neighborly constant $\delta$. Hence if $\delta<$ $\varepsilon / 4 c^{2}$, the space $Z$ will have the $\left(\varepsilon / c^{2}, \delta\right)$-neighborly tree property. In general, however, the $(\varepsilon, \delta)$-NTP is not an isomorphic invariant since the condition $\delta<\varepsilon / 4$ is essential. In fact, the spaces $X_{\delta}$ that are constructed in [2] have the $(2, \delta)$-NTP and are all isomorphic to subspaces of $l_{1}$ that cannot possess the $(\varepsilon, \delta)$-NTP for any $\varepsilon$ and $\delta$. On the other hand, it follows from the above argument that if a space $X$ has the $(\varepsilon, \delta)$-NTP for a given $\varepsilon>0$ and every $\delta<\varepsilon / 4$, then any isomorphic image of $X$ will have the $\left(\varepsilon^{\prime}, \delta\right)$-NTP for an appropriate $\varepsilon^{\prime}>0$ and every $\delta<\varepsilon^{\prime} / 4$.

EXAMPLe. A separable Banach space $X$ with the RNP that is not isomorphic to a subspace of a separable dual space.

From Example 9 in [2], for each $n \geqslant 3$ there exists a separable Banach space $X_{n}$ with the RNP and the $(2,1 / n)$-NTP. Let $X=\left(\Sigma \oplus X_{n}\right)_{1}$ be the $l_{1}$ product of the $X_{n}$.

Then $X$ is a separable Banach space that has the RNP since each of the spaces $X_{n}$ has the RNP [1, p. 219]. Also, for any $n$ the space $X_{n}$ is contained in $X$ via the natural isometric embedding and so the space $X$ has the $(2,1 / n)$ NTP for every $n \geqslant 3$. From our remarks above it follows that if $\varphi: X \rightarrow Z$ is an isomorphism of $X$ into a dual Banach space $Z$, then $Z$ will have the $(\varepsilon, \delta)$-NTP for some $\varepsilon$ and $\delta$. But then by Theorem 6 in [2], $Z$ contains a tree and so cannot have the RNP. Since any separable dual space has the RNP, it follows that $X$ is not isomorphic to a subspace of a separable dual.

REMARK. While preparing this note for publication, the authors were informed that J. Bourgain and F. Delbaen, $A$ special class of $\mathcal{L}_{\infty}$ spaces have independently obtained a counterexample to Uhl's conjecture.

\section{BIBLIOGRAPHY}

1. J. Diestel and J. J. Uhl, Jr., Vector measures, Math. Surveys, no. 15, Amer. Math. Soc., Providence, R. I., 1977.

2. P. W. McCartney, Neighborly bushes and the Radon-Nikodym property for Banach spaces, Pacific J. Math. (to appear). 
3. On some Banach space properties related to the Radon-Nikodjm property, Ph.D. Dissertation, Claremont Graduate School, 1978.

4. C. Stegall, The Radon-Nikodym property in conjugate Banach spaces, Trans. Amer. Math. Soc. 206 (1975), 213-223.

5. J. J. Uhl, Jr., A note on the Radon-Nikody'm property for Banach spaces, Rev. Roumaine Math. Pures Appl. 17 (1972), 113-115.

Department of Mathematical Sciences, Northern Kentucky University, Highland HeioHTS, KeNTUCKY 41076 